\title{
Choosing the preventive workload in general practice: practical application of the Coronary Prevention Group guidelines and Dundee coronary risk-disk
}

\author{
Tony Randall, John Muir, David Mant
}

\section{Abstract}

Objective-To determine the workload implications for general practice of the Coronary Prevention Group and British Heart Foundation action plan for preventing heart disease.

Design-Computer simulation of plan, including calculation of Dundee risk scores, with data from OXCHECK trial.

Subjects -4759 patients aged 35-64 who had health checks during 1989-91.

Main outcome measure-Effect of using different risk scores as thresholds on workload and coverage of patients at known risk. Thresholds of 6-20 were used for cholesterol screening (nearset) and 4-16 for special care (preset).

Results-On the basis of workload a nearset of 8 and preset of 12 would be reasonable. This implies cholesterol measurement in $1794(37.7 \%)$ patients and special care in $1074(22 \cdot 6 \%)$. However, many patients with single risk factors were not allocated to special care at these thresholds: $11(37.9 \%)$ patients with cholesterol concentrations $\geqslant 10 \mathrm{mmol} / \mathrm{l}, 21$ (33.9\%) with systolic pressure $\geqslant 180 \mathrm{~mm} \mathrm{Hg}$, and $213(40.7 \%)$ heavy smokers ( $>20$ cigarettes/day) were missed. The distribution of scores was similar in those at established clinical risk, those with family history of heart disease, and others.

Conclusion-The guidelines may help to make best use of resources within specific age-sex groups but sound protocols for unifactorial risk assessment and modification remain essential.

\section{Introduction}

The Coronary Prevention Group and British Heart Foundation recently published guidelines on preventive care in general practice based on formal risk assessment with the Dundee coronary risk-disk. '? These guidelines recognise that resources are limited and suggest how practices should set priorities in preventing coronary heart disease by choosing which patients need special care and which can be given general advice. Figure 1 shows the key decisions. The first step is to measure blood pressure and record smoking habit, personal medical history (of ischaemic heart disease, diabetes, hypertension, or hyperlipidaemia), and close family history (of premature heart disease before the age of 50 years or familial hyperlipidaemia) in all patients aged 18-74 years. This process divides the population into three groups: those with established disease (clinical risk group), those with a family history but no established disease (family risk group), and the remainder (general risk group). All people in the clinical and family risk groups have cholesterol concentrations measured. All those with clinical risk are allocated to special care. Allocation of those at family risk to special care depends on their

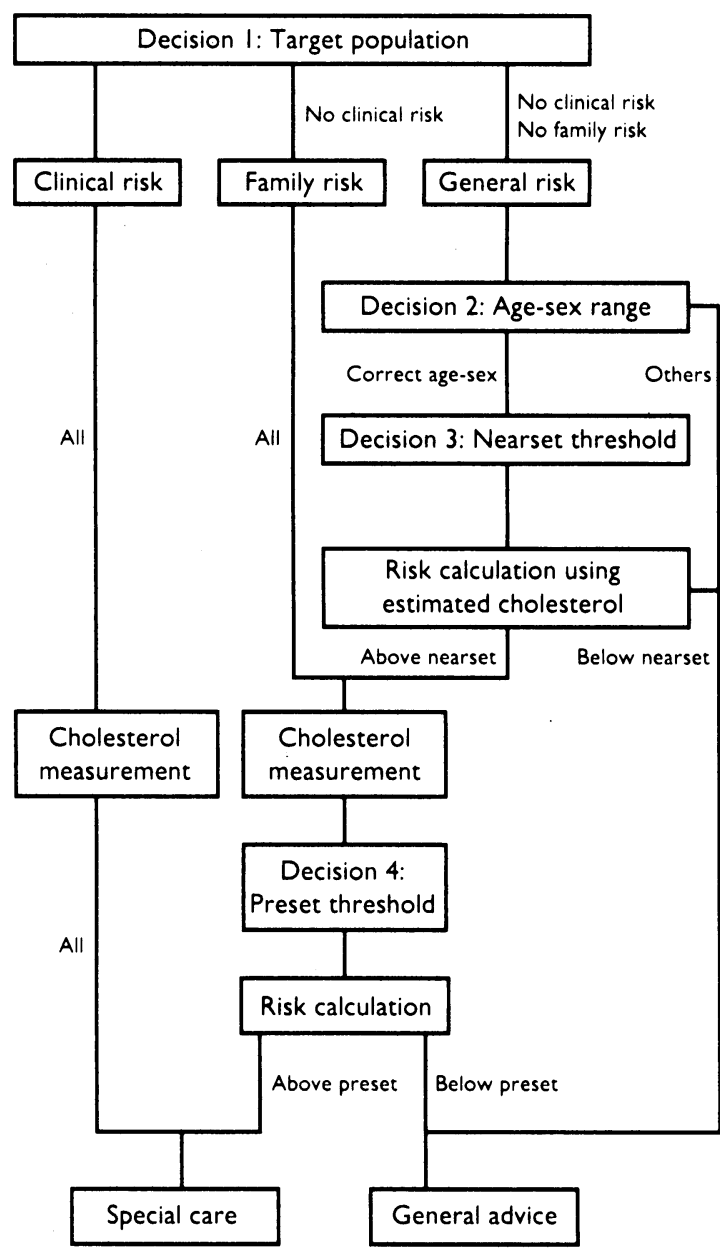

FIG 1-Coronary Prevention Group guidelines: decision flowchart

overall Dundee risk score: only those above a given threshold (preset) are selected.

The more complicated part of the guidelines deals with the allocation of patients at general risk to special care. There are three stages in this process. Firstly, some patients within the general risk group are excluded from further consideration for cholesterol measurement or special care because they fall outside a predetermined age and sex range. Secondly, some of the remaining patients are excluded because their estimated Dundee score, calculated from the population mean total cholesterol concentration for the specific age-sex group, is below a defined cut off point (the nearset). Thirdly, total cholesterol is measured in a blood sample and only those above the preset threshold when their actual Dundee risk score is recalculated are allocated to special care.

The guidelines give only limited information about the practical implications of making these different 
decisions. Practices must know how many patients will be identified for special care, how many will need cholesterol concentration measured, and how many patients at generally accepted high risk (for example, heavy smokers) will receive only general advice when specific thresholds are chosen and specific groups screened. We therefore decided to write a computer simulation to apply the guidelines to about 5000 patients screened in the OXCHECK trial in 1989-91. OXCHECK is a randomised controlled trial of health checks in patients aged 35-64 years from five general practices in Bedfordshire. 'The uptake of screening has been high (about $80 \%$ ) and the trial provides a useful estimate of the overall prevalence of cardiovascular risk in a general practice population. The practical outcome in the OXCHECK population of making the different decisions required by the working group's guidelines are described and some modifications suggested.

\section{Methods}

Details of the methods of the OXCHECK trial have been described. ${ }^{3}$ Briefly, the trial is based in five general practices in Luton and Dunstable, Bedfordshire. In late 1988 a computerised list was obtained from the family practitioner committee of all patients registered with these practices who would be aged 35-64 years on 1 January 1989. A questionnaire was sent to 17965 patients and an adjusted response rate obtained of $80.3 \%$. Respondents were randomised to be offered a health check during one of four years beginning in June 1989. The acceptance rate since the beginning of the study has been about $80 \%$. At the health check, details of personal and family history of ischaemic heart disease, stroke, hypertension, diabetes, and cancer were recorded and a history of smoking habits was taken. Blood pressure was measured according to standard protocols with a Hawksley random zero sphygmomanometer. Cholesterol concentration was measured in serum samples by using the cholesterol oxidase/peroxidase-aminopyrine method with an Olympus AU5000 autoanalyser. The main outcome measures for the trial are the mean levels of individual risk factors, and these will be reported in 1993.

The simulation was written with the d-base dialect Clipper and was run on a 386 IBM compatible

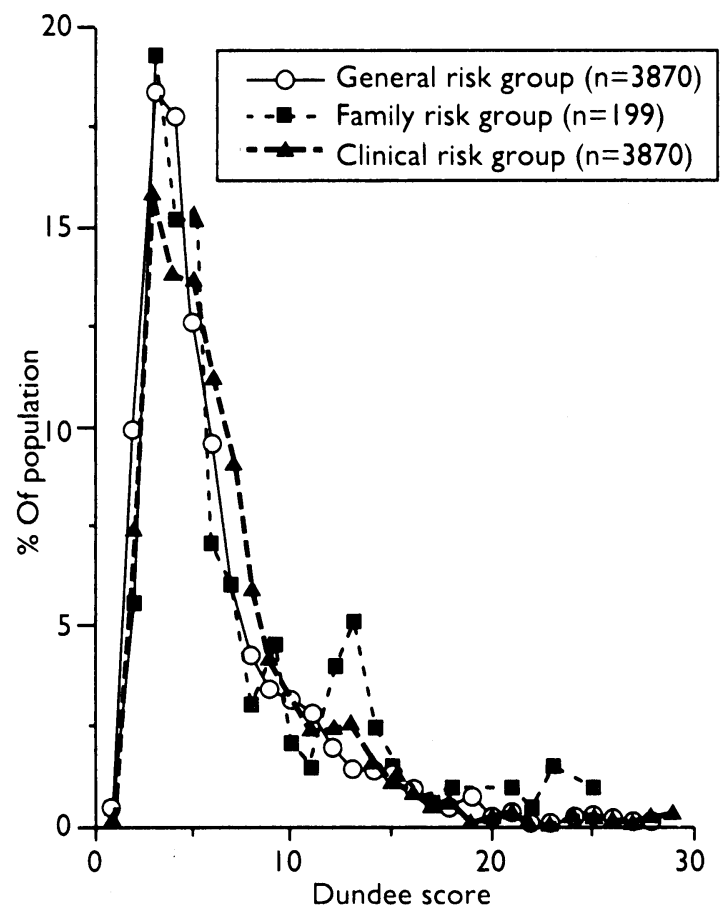

microcomputer. All 5097 patients who had had a health check before 1 October 1991 were included, except for 338 patients in whom results were not complete (usually because of loss or breakage of blood specimens) or who were aged over 65 years at the time of their health check. This left a total of 4759 patients, 2086 men and 2673 women, to be included in the simulation. The data items used were identical to those suggested by the working group except for previously established hyperlipidaemia and associated signs, which are not routinely available in OXCHECK. The size of the clinical risk group will therefore be slightly underestimated, although the effect of this on the results is likely to be small. The definition used foro family history of coronary heart disease was any firsto degree relative in whom disease was diagnosed before $\frac{\bar{p}}{\bar{b}}$ the age of 50 years. The calculation of the Dundee risk $\underset{\propto}{\mathbb{\Phi}}$ score was straightforward except for smoking history, for which the OXCHECK data do not include the number of cigarettes smoked by former smokers. The. assumption was therefore made that former smokers $\overrightarrow{-}$ had smoked 10 cigarettes a day. The Dundee scores calculated for the simulation were checked against the음 result obtained with Dundee risk-disk computer software supplied by Professor Tunstall-Pedoe.

The results of the simulation are presented in terms: of the risk score rather than the risk rank. This is because the rank is dependent on the population. distribution of risk, which is clearly different in the N OXCHECK population from that in the Scottish ${ }^{V}$ population used to derive the Dundee rank: risk scores 5 of $20,16,12,8$, and 6 correspond to Dundee ranks N of about $5,10,20,35$, and 50 respectively and $ᄃ$ OXCHECK ranks of $2 \cdot 5,5,11,23$, and 37 . Basing $₹$ workload estimates on the Dundee rank would there- $\vec{\bullet}$ fore be very misleading. Confidence intervals were calculated with the CIA programme. ${ }^{+}$

\section{Results}

Dundee score distribution-Figure 2 shows the frequency distribution of the Dundee score in the OXCHECK population for the clinical risk, familyô risk, and general risk groups. The similarity in distri- 3 bution is striking (and unexpected). In all groups the distribution is heavily skewed. Most patients scored? less than 5 (the median was between 4 and 5) and there was a long tail (the highest score recorded was 84 ). The Dundee score is directly proportional to risk, soo everyone in the tail with a score of 20 or more is at least $\stackrel{3}{\text {. }}$. four times as likely as the person with the median scoreo (of similar age and sex) to have a cardiovascular event. General practitioners are unlikely to want to excludeo these high risk patients by setting a preset threshold above 20 or to try to offer special care to more than half their patients by selecting a threshold of 5 or less. Hence the preset thresholds reported in this paper are in the range 6 to 20 .

Clinical and family risk - Table I shows the propor- $-\omega$ tion of patients in each age-sex group at establishedo clinical risk or at family risk. The working groupo guidelines suggest that all patients with established clinical and family risk should have their cholesterol?

TABLE I-Numbers (percentages) of patients with established clinical risk and reported family risk according to sex and age (years,

\begin{tabular}{|c|c|c|c|}
\hline & $\begin{array}{c}\text { No of } \\
\text { patients }\end{array}$ & $\begin{array}{l}\text { No }(\%) \text { with } \\
\text { clinical risk }\end{array}$ & $\begin{array}{l}\text { No (\%) with } \\
\text { family risk }\end{array}$ \\
\hline \multicolumn{4}{|l|}{ Men: } \\
\hline $35-44$ & 647 & $25(3.9)$ & $36(5 \cdot 6)$ \\
\hline $45-54$ & 728 & $87(12 \cdot 0)$ & $35(4.8)$ \\
\hline $55-64$ & 711 & $190(26 \cdot 7)$ & $31(4 \cdot 4)$ \\
\hline \multicolumn{4}{|l|}{ Women: } \\
\hline 35.44 & 872 & $51(5 \cdot 9)$ & $50(5 \cdot 7)$ \\
\hline $45-54$ & 975 & $103(10 \cdot 6)$ & $55(5 \cdot 6)$ \\
\hline $55-64$ & 826 & $234(28 \cdot 3)$ & $36(4 \cdot 4)$ \\
\hline
\end{tabular}

FIG 2-Distribution of Dundee score in clinical, family, and general risk groups 


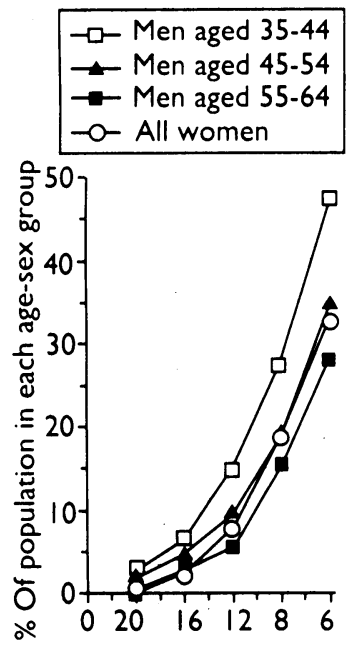

$(16)(12)(8)(6)(4)$

FIG 3-Cumulative percentage of patients at general risk assigned to special care according to Dundee score thresholds (thresholds for cholesterol screening in parentheses)

FIG 4-Cumulative percentage of each group in whom cholesterol would be measured because of clinical risk, family risk, and different nearset thresholds in those at general risk concentration measured and that all the patients with clinical risk be assigned to special care. If these guidelines are followed, by the age of 55 more than a quarter of the population would be allocated to special care because of established clinical risk. There was surprisingly little difference in the level of established clinical risk between men and women. The family risk group includes only $199(5 \cdot 1 \%, 95 \%$ confidence interval $4.5 \%$ to $5 \cdot 7 \%$ ) of the total population, and this proportion varies little with age or sex. The cumulative number of patients with family risk allocated to special care according to Dundee score presets of $20,16,12,8$ and 6 are $10(5 \cdot 0 \%), 15(7 \cdot 5 \%), 41(20 \cdot 6 \%), 63(31 \cdot 7 \%)$, and $89(44 \cdot 7 \%)$ respectively.
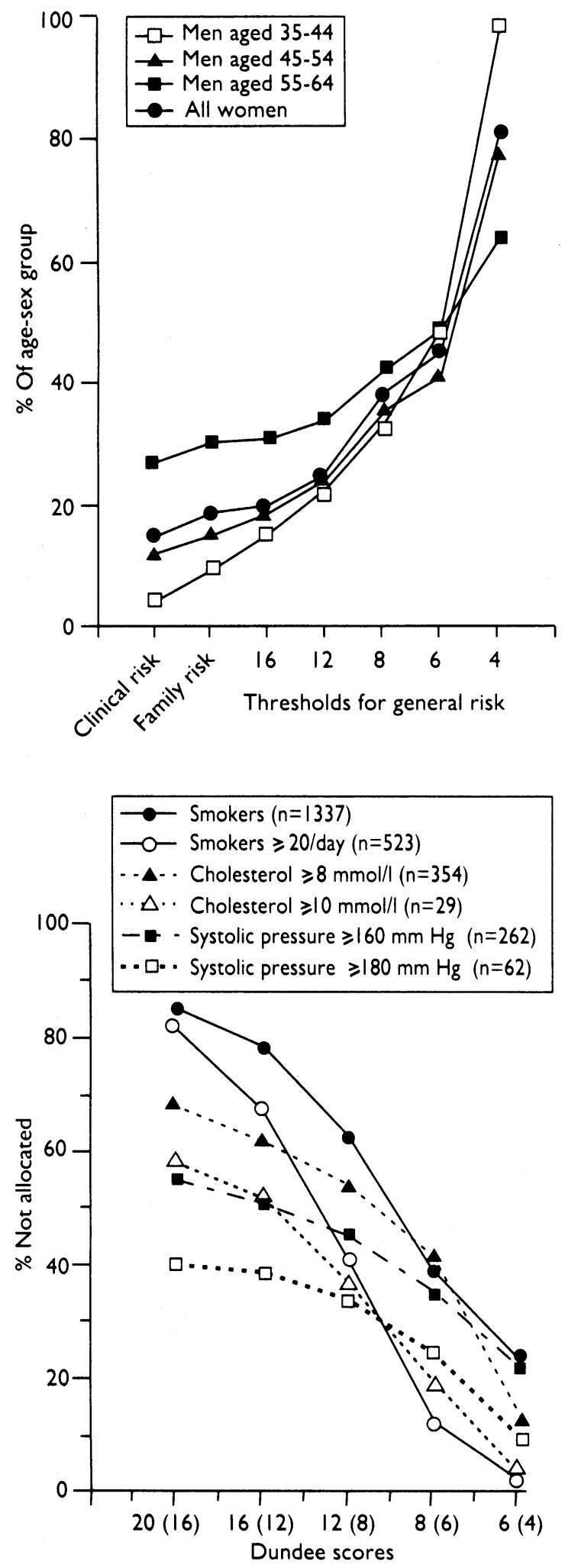

General risk-The allocation of patients at general risk to special care according to different presets is shown in figure 3 . In women there was little difference between the age groups in the proportion assigned to special care and so the results are not broken down by age. A preset threshold of 12 for women assigns 174 $(8.0 \%, 6.9 \%$ to $9 \cdot 2 \%)$ to special care and a threshold of 6 assigns $711(32 \cdot 7 \%, 30 \cdot 8 \%$ to $34 \cdot 7 \%)$. In men, the proportion assigned to special care at each cut off level decreases with age: a threshold of 12 assigns $87(14.8 \%$, $11.9 \%$ to $17 \cdot 7 \%)$ of those aged $35-44$ and $28(5.7 \%$, $3.8 \%$ to $8 \cdot 1 \%$ ) of those aged $55-64$. A threshold of 6 assigns $280(47 \cdot 7 \%, 43 \cdot 7 \%$ to $51 \cdot 7 \%)$ of those aged $35-44$ and $139(28 \cdot 0 \%, 24 \cdot 1 \%$ to $32 \cdot 0 \%)$ of those aged 55-64 to special care. In determining the overall number of patients assigned to special care according to the Coronary Prevention Group guidelines these proportions must be added to those already assigned $\omega$ because of clinical and family risk. The higher propor- $\vec{O}$ tion of young men assigned from the general risk group is counterbalanced by the lower proportion of this group at clinical risk.

Cholesterol measurement-The guidelines suggest 3 . that total cholesterol concentrations should be $\omega$ measured in all patients with family risk and clinical or risk, but only in those with general risk who have a $\circ$ Dundee risk score above a given threshold on the basis $\vec{v}$ of the estimated total cholesterol. The guidelines also N suggest that the nearset threshold should be below the $v$ preset adopted for allocation to special care. Figure 4 윽 shows the proportion of people in whom cholesterol $N$ would be measured on the basis of different nearset $c$ thresholds in the general risk group. Women are again not subdivided by age (although the size of the clinical $\vec{\theta}$ risk group does vary by age in women (table I)). With a nearset threshold of 8 , which might be used with a preset level of 12,1022 women $(38 \cdot 2 \%, 36 \cdot 4 \%$ to $40 \cdot 1 \%), 217$ men aged $35-44(33 \cdot 5 \%, 29 \cdot 9 \%$ to $37 \cdot 2 \%)$, and 297 men aged $55-64(41 \cdot 8 \%, 38 \cdot 1 \%$ to $45 \cdot 4 \%)$ would be offered blood cholesterol measurement. The effect of the nearset threshold can be seen by com- $\stackrel{\Phi}{\square}$ paring the distribution of actual Dundee scores in the $\overrightarrow{\overrightarrow{0}}$ general risk group (fig 2) with the number allocated to 3 special care with specific presets (fig 3). The nearset threshold makes a greater difference if high preset levels are chosen. At a preset of 20 only 34 of the 90 $(37.8 \%)$ patients with an actual Dundee score of 20 or more were selected for special care. This is because the nearset was 16 and cholesterol concentration was not 3 measured in 56 patients with an estimated score below 16 but a cholesterol concentration high enough to increase their score to 20 . In contrast, at a preset of 6 , 0 1342 of the $1582(84.8 \%)$ patients with an actual score of 6 were selected, even though a closer nearset of 4 was 0 used.

Patients at high unifactorial risk-One problem with the multifactorial approach to risk estimation is that $N$ some people at high unifactorial risk will be missed and $\underset{\mathrm{W}}{\mathcal{N}}$ may not be allocated to special care. Figure 5 therefore $O$ looks at the proportion of all people at high unifactorial 0 risk who are not assigned to special care when different presets are applied to the general and family risk $\stackrel{\mathcal{D}}{?}$ groups. At a preset of 12 (nearset 8), $213(40 \cdot 7 \%$, 무 $36.5 \%$ to $44.9 \%$ ) smokers of 20 or more cigarettes a day, $21(33 \cdot 9 \%, 22 \cdot 3 \%$ to $47 \cdot 0 \%)$ of those with systolic $\stackrel{D}{\Omega}$ blood pressure $\geqslant 180 \mathrm{~mm} \mathrm{Hg}$, and $11(37 \cdot 9 \%, 20 \cdot 7 \%$ to $\mathbb{D}$ $57.7 \%$ ) of those with a total cholesterol concentration $\geqslant 10 \mathrm{mmol} / \mathrm{l}$ would be allocated to general advice rather than to special care, even though all patients with clinical risk were so allocated irrespective of Dundee score.

Overall workload-Table II shows the overall proportion of all patients aged 35-64 attending for a health check who, according to the guidelines, would need to be offered cholesterol measurement and who would be 
TABLE II - Proportion of all patients aged 35-64 years $n=4759$, having cholesterol measured and allocated to special care according to specific nearset and preset values

\begin{tabular}{|c|c|c|c|c|c|}
\hline & \multicolumn{5}{|c|}{ Nearset preset } \\
\hline & $16(20)$ & $12(16)$ & $8(12)$ & $6(8)$ & $4(6)$ \\
\hline $\begin{array}{l}\text { Cholesterol measurement }{ }^{\star}: \\
\text { No }(\%) \\
95 \% \text { Confidence interval }(\%) \\
\text { Special care: }\end{array}$ & $\begin{array}{r}980(20 \cdot 6) \\
19 \cdot 4 \text { to } 21 \cdot 7\end{array}$ & $\begin{array}{l}1219(25 \cdot 6) \\
24 \cdot 4 \text { to } 26 \cdot 9\end{array}$ & $\begin{array}{l}1794(37 \cdot 7) \\
36 \cdot 3 \text { to } 39 \cdot 1\end{array}$ & $\begin{array}{l}2155(45 \cdot 3) \\
43 \cdot 9 \text { to } 46 \cdot 7\end{array}$ & $\begin{array}{l}3813(80 \cdot 1) \\
79 \cdot 0 \text { to } 81 \cdot 3\end{array}$ \\
\hline $\begin{array}{l}\text { No }(\%) \\
95 \% \text { Confidence interval }(\%)\end{array}$ & $\begin{array}{r}734(15 \cdot 4) \\
14 \cdot 4 \text { to } 16 \cdot 4\end{array}$ & $\begin{array}{r}831(17 \cdot 5) \\
16 \cdot 4 \text { to } 18 \cdot 5\end{array}$ & $\begin{array}{l}1074: 22 \cdot 6 \\
21 \cdot 4 \text { to } 23 \cdot 8\end{array}$ & $\begin{array}{l}1512(31 \cdot 8) \\
30 \cdot 4 \text { to } 33 \cdot 1\end{array}$ & $\begin{array}{l}2112(44 \cdot 4) \\
43.0 \text { to } 45 \cdot 8\end{array}$ \\
\hline
\end{tabular}

allocated to special care on the basis of specific preset and nearset thresholds.

\section{Discussion}

There is a recognised need to set priorities for prevention of cardiovascular disease in general practice. The potential workload is immense, and the Coronary Prevention Group and the British Heart Foundation guidelines (using the Dundee risk-disk) have much to recommend them. They take account of the synergistic interaction of risk factors, they recognise that many patients already have established clinical conditions such as hypertension, and they suggest a system for rationing cholesterol measurement and for targeting follow up resources where they are likely to do most good. However, the simulation raised several practical problems in the application of the guidelines. Many problems arise from ambiguity rather than more serious difficulties, but we suspect that other general practitioners share our confusion and will value clarification.

\section{DRAWBACKS OF GUIDELINES}

The most obvious problem is the number of people at high risk from one factor but at low risk from all others. Such people may have low Dundee scores but be at high risk of disease. This is because risk factors such as smoking or hypertension confer risk for diseases other than coronary atheroma and because at the extremes of risk the data on which the multifactorial model was built are scanty. For example, the weighting for cholesterol concentration does not reflect the high risk of young people with familial hyperlipidaemia (recently reported as associated with a mortality almost 100 times that expected at ages $20-39) .5$ At a preset threshold of 12 , our results show (fig 5) that a quarter of heavy smokers and more than a third of patients with notably raised blood pressure and total cholesterol concentrations are likely to be allocated to the general advice group. Although it is unclear what exactly is meant by special care compared with general advice, and the authors of the guidelines state that "attention to special care should not diminish existing effort to advise all smokers, people with borderline hypertension and all those who are overweight," this issue of unifactorial risk must be dealt with specifically. The clinical priority is to establish clear protocols for the follow up of each individual risk factor, particularly hypertension, diabetes, and hyperlipidaemia. These protocols must be applied to all patients screened and will define those who need special clinical care. This will include people with borderline hypertension, mild hyperlipidaemia, and impaired glucose tolerance as well as those with established clinical disease.

Several other practical issues raised by the working group's algorithm (fig 1) need clarification. Firstly, the flow chart suggests that all patients aged 18 to 74 years should be screened but that risk calculation should be performed only on certain subgroups defined by age and sex. Selective calculation of risk score seems unnecessary. All of the data required to make the calculation (assuming use of estimated cholesterol concentration) will be available from the initial health assessment. Secondly, the Dundee score does not adjust for the absolute differences in risk with age and between men and women. This does not imply selective calculation of score by age and sex but means that the disk is best used to allocate resources within a specific age-sex group, after taking account of the absolute risk and the effectiveness of intervention in each group. Thirdly, we disagree with the advice that the Dundee rank is more useful than the Dundee score. The score is directly proportional to relative risk and can be interpreted in relation to a median population score of about 5 . The use of the rank is promoted to give a clear indication of the work generated by adopting different thresholds, but the distribution of the score is clearly different in different populations. Thus the rank shown on the disk can be misleading in predicting workload. Choosing a threshold for special care of Dundee rank 20 includes only $11 \%$ of the OXCHECK population - which overestimates workload by almost $100 \%$. In giving personal advice an error may also arise from the fact that the risk-disk weightings are based on data for 40-59 year old men screened between 18 and 20 years ago. ${ }^{6}$

As a proposed mechanism for rationing cholesterol measurement, the guidelines are undoubtedly contentious. As previously reported, family history is not a good predictor of high total cholesterol concentration in the OXCHECK data. ${ }^{3}$ We remain unconvinced in the light of these data and those of the national lipid screening study that selective testing on the basis of family history, clinical history, or clinical signs is empirically justified if the main objective is identification of the $0.5 \%$ of patients at high risk because of specific problems of lipid metabolism. A significant number of patients with total cholesterol concentrations $\geqslant 10 \mathrm{mmol} / \mathrm{l}$ will not be detected unless screening thresholds are used that would require measuring cholesterol in most patients. However, universal screening is not attractive. The pressure of the pharmaceutical industry and the wish of patients and colleagues to deal with risk detected make it likely that many patients with moderately raised cholesterol concentrations will be inappropriately treated with drugs. It therefore seems that, on pragmatic grounds, the concept of measuring cholesterol only in those at high overall risk should be supported.

\section{CONCLUSIONS}

Our practical conclusions for general practitioners as a result of our simulated attempt to apply the guidelines to a screened middle aged population are as follows. The first priority remains the clinical assessment and follow up of individual risk factors, irrespective of overall risk. This requires special care in the sense of well defined and audited follow up of smokers and patients with mild hypertension, moderate hypercholesterolaemia, impaired glucose tolerance, and obesity. The Dundee risk score should be used to decide which patients merit priority for intensive lifestyle intervention within specific age-sex groups The choice of which age-sex groups merit priority depends on the primary criteria of absolute risk and established effectiveness of intervention in each group. Patients with family risk do not need to be handled differently from other patients. The Dundee risk score should be calculated in all patients. Few practices could cope effectively with the workload generated by setting a screening threshold below 8 or a special care threshold below 12 .

We thank Pat Yudkin for advice on statistical analysis and data presentation and the OXCHECK study group for permission to use OXCHECK data in this simulation. 
OXCHECK is funded primarily by the Imperial Cancer Research Fund.

1 Working Group of the Coronary Prevention Group and the British Heart Foundation. An action plan for preventing coronary heart disease in primary care. BMF 1991;303:748-50.

2 Tunstall-Pedoe $H$. The Dundee coronary risk-disk for management of change in risk factors. BMF 1991:303:744-7.

3 Imperial Cancer Research Fund OXCHECK Study Group. Prevalence of risk factors for heart disease in OXCHECK trial: implications for screening in primary care. $B M \mathcal{F}$ 1991;302:1057-60.
+ CIA [compurer program] London: BMJ, 1989.

Scientific Committee of the Simon Broom Register Group. Risk of tat coronary heart disease in familial hvpercholesterolaemia. B.11 1991:303 $893-6$

6 World Health Organisation European Collaborative Group. Multifactorial tria in the prevention of coronary heart disease. 1 . Recruitment and initia findings. Eur Heart $\mathcal{f}$ 1980:1:73-80

7 Mann J, Lewis B, Shepherd J, Winder A, Fenster S, Rose L, et al. Blood lipid concentrations and other cardiovascular risk factors: distribution, prevalence, and detection in Britain. BMF 1988;296:1702-5.

Accepted I fuly 1992

\title{
Value of the Dundee coronary risk-disk: a defence
}

\author{
Hugh Tunstall-Pedoe
}

We invited Professor Tunstall-Pedoe, who is a member of the Coronary Prevention Group and originator of the Dundee coronary risk-disk, to comment on the results of the $O x$ ford computer simulation.

\section{Cardiovascular \\ Epidemiology Unit,} Ninewells Hospital and Medical School, Dundee DD1 9SY

Hugh Tunstall-Pedoe, director

BMF 1992;305:231-2
We launched the "Action Plan" and Dundee coronary risk-disk ${ }^{2}$ in September 1991. So far 3500 disks have been purchased. We recently surveyed users, and their opinions, from clinical practice, contrast with the judgments from Randall et al's modelling exercise.

The action plan and risk-disk were designed not just to categorise risk but to motivate change and monitor progress. The Dundee rank places patients from the front (one) to the back (100) of a metaphorical bus queue for a heart attack. ${ }^{2}$ The ranking is based on the Dundee score, which combines smoking, blood pressure, and serum cholesterol concentration, and on the distribution of that score in a large random population survey, the Scottish heart health study. ${ }^{3}$

The Dundee rank has been welcomed because the bus queue metaphor and 1-100 frame of reference are understood. In rejecting the Dundee rank in favour of the Dundee score Randall et al have been precipitate. They are at odds with most users, and rewriting of the action plan using the Dundee score may cause unnecessary difficulty for readers. They are not only throwing out the baby with the bath water but are also going to miss the bus.

\section{User survey}

The British Heart Foundation sent a questionnaire to 296 randomly selected purchasers of the Dundee coronary risk-disk. We have had 187 respondents, 156 of whom had used the disk on some 10000 patients but with great variation. The 31 others had not opened the packet; had not learnt to use it; had experienced difficulties (lessened in those watching the instructional video); or had not bought the disk for themselves. Many responses were incomplete, but questions relevant to Randall et al's paper follow.

Asked about use of a protocol, 28 did not answer, 70 used no protocol, 52 used a practice protocol, and six used the Coronary Prevention Group and British Heart Foundation action plan' (which might have influenced some of the other protocols). Twenty two respondents did not state whether they showed the risk calculations to patients, but 74 always did, and 49 sometimes did; only 11 never did. Twenty seven did not state a preference for the Dundee rank or score, but 57 preferred the rank, 31 preferred the score, and 41 used both equally. Twenty five did not state whether they thought that the disk motivated patients, 130 thought that it did (57 answered an unqualified yes and 73 answered sometimes), and only one respondent thought it did not. Asked whether they would recommend the Dundee coronary risk-disk to others, nine did not answer, six ticked no, and 141 ticked yes (49 without reservation and 92 with qualification). Sixty two respondents said they would attend user meetings. Most responses were confined to the codings, but additional comments were received from 52 , explaining non-use (as above) or enthusiasm (see box).

\section{Users' comments on Dundee coronary risk-disk}

Family doctor (used on 100 patients): This is an excellent development. The ability to numerically tell patients how much they can achieve by changing their risk parameters is very motivating.

Practice nurse (50 patients): The risk-disk has been very useful for assessment and made my job more interesting and the patients do seem interested also.

Occupational physician (500 patients): It works well. It motivates patients by figures they can understand and not by rhetoric from the doctors.

Family doctor (200 patients): I find the disk excellent. Multifactorial risk assessment remains hopelessly vague without some form of complicated calculation which the disk performs so easily. Most patients understand and appreciate the "queuing for a coronary" analogy and undoubtedly the feedback they obtain about moving down the queue following lifestyle change reinforces motivation. I am often surprised at the rank result compared with my own subjective risk assessment and this saves me considerable time by avoiding excessive effort in low risk people. I know four recidivist smokers who have given up (for 2-8 months so far) once they found they were at the front of the queue.

\section{OXCHECK and Scottish data}

Randall $e t$ al rejected the Dundee rank because of the unexplained difference in distributions of risk score in OXCHECK and the Scottish heart health study. ${ }^{3}$ Practice populations may differ because smoking varies by social class, and there are potential biases in other factors. This does not invalidate the Dundee rank as an external, population based frame of reference. The action plan suggested that each practice adjusts its cut off points from experience in its own population.' Relative rankings are unaffected by adjusting the scale.

Randall et al imply that because the OXCHECK and Scottish data disagree, the Scottish data are misleading. However, 10000 Scottish men and women were studied from 260 practices in 22 districts across Scotland by using international standardisation and quality control, and the results are published. ${ }^{3}$ The OXCHECK data come from 5000 patients in five clustered practices in one corner of southern England and their full risk factor data are not published. ${ }^{+}$The large and puzzling deviation between the OXCHECK and Scottish studies cannot be explained until the OXCHECK data are released; meanwhile there is no inherent reason to consider OXCHECK cut off points a better guide for British doctors than the Scottish study and the Dundee rank. Recent British surveys show insignificant differences in risk factors between Scotland and England." 